\title{
Effects of Structured Nontarget Stimuli on Saccadic Latency
}

\author{
Brian J. White, Karl R. Gegenfurtner, and Dirk Kerzel \\ Justus-Liebig-Universität, Allgemeine Psychologie, Giessen, Germany
}

Submitted 22 October 2004; accepted in final form 27 January 2005

White, Brian J., Karl R. Gegenfurtner, and Dirk Kerzel. Effects of structured nontarget stimuli on saccadic latency. $J$ Neurophysiol 93: 3214-3223, 2005. First published February 2, 2005; doi:10.1152/jn.01104.2004. It has been suggested that the remote distractor effect is the result of nontarget stimulation of a central region representing a collicular fixation zone near the time of target onset. The distributed network of the cells responsible for this effect is believed to extend over a large area, responding to distractors $\leq 10 \mathrm{deg}$ in the periphery. Several studies also implicate the superior colliculus as the substrate behind an inhibited saccadic response arising from a display change. We investigated this further by using a patch of pink noise of various sizes as a nontarget stimulus. We show that the onset of a small patch $(2.3 \times 2.3 \mathrm{deg})$ of centrally displayed pink noise can produce a significant increase in saccadic latency to a simultaneously presented peripheral Gabor target. In contrast, a large patch $(36 \times 36 \mathrm{deg})$ of pink noise did not increase latency despite the fact that it also stimulated the region representing the fixation zone. Furthermore, only the large patch of noise facilitated latency when presented before target onset. We also examined the effect of patch sizes between these two extremes and found a steady decrease in latency as patch size increased. This confirms that nontarget stimulation of the region representing the fixation zone near the time of target onset is not in itself sufficient to produce the increase in latency typically found with remote distractors. The results are consistent with the idea that only a spatially confined object leads to a discharge of collicular fixation neurons.

\section{N T R O D U C T I O N}

Saccadic eye movements rapidly center a peripheral target at the retinal position of highest acuity, the fovea. The latency at which this occurs is highly variable and is affected by specific experimental conditions. It is well known, for example, that the offset of a fixation point 100 to $200 \mathrm{~ms}$ before the onset of a target can significantly reduce saccadic latencies ("gap effect"; Saslow 1967), and under certain circumstances can produce a bimodal distribution of response times, the first mode being very fast with a peak around $100 \mathrm{~ms}$ ("express saccades"; Fischer and Ramsperger 1984).

In contrast, the onset of a nontarget stimulus near the time of target onset can increase saccadic latency and eliminate express saccades (Braun and Breitmeyer 1990; Lévy-Schoen 1969; Olivier et al. 1999; Walker et al. 1995, 1997; Weber and Fischer 1994). This "distractor effect" was most often reported with a nontarget stimulus appearing at the mirror-symmetric opposite hemifield of the target, but it is also known to occur at various spatial locations (Walker et al. 1997), with multiple distractors (Weber and Fischer 1994), and more recently with respect to smooth pursuit latency (Knox and Bekkour 2004). Weber and Fischer (1994), for example, measured the propor-

\footnotetext{
Address for reprint requests and other correspondence: B. J. White, Justus-LiebigUniversität Giessen, Abteilung Allgemeine Psychologie, Otto-Behaghel-Strasse 10F, D-35394 Giessen, Germany (E-mail: brian.j.white@ psychol.uni-giessen.de).
}

tion of express saccades (in a gap paradigm) to an oriented target line $4.5 \mathrm{deg}$ to the right of fixation during the simultaneous onset of a large number of distractors of another orientation. The result was a pop-out display containing about $23 \times$ 30 items, extending 15 deg vertically by 20 deg horizontally. Weber and Fischer found that express saccades were eliminated in this case. They then compared this to a condition in which only 2 distractors appeared foveally $(0.5 \mathrm{deg}$ on either side of fixation) and found nearly the same reduction in express saccades. They explained the results in relation to the so-called dead zone for express saccades (Weber et al. 1992), a finding in which express saccades are eliminated to targets appearing within the central 2 deg visual angle. Along these lines, Weber and Fischer (1994) suggested that nontarget stimuli appearing in this region might also inhibit rapid saccades to a peripheral target by reactivating foveal attention after its prior disengagement from the offset of the fixation point.

The superior colliculus (SC) is believed to be an important neurological substrate underlying several known effects on saccadic latency (Munoz and Wurtz 1993b, 1995a), in particular the gap effect, but also the remote distractor effect (Findlay and Walker 1999; Walker et al. 1997). This structure is retinotopically organized, and cells at the rostral end (a region extending approximately 2 deg foveally) are known to play a significant role in visual fixation (Munoz and Wurtz 1993a). Thus this region was termed the fixation zone. Cells here show increased firing rates during active fixation and pause during saccades. This pause is believed to be necessary for saccade generation, and the removal of a visual fixation stimulus causes a break in activity that results in the latency decrease seen during a gap paradigm (Munoz and Wurtz 1993b, 1995a). Throughout the remainder of the intermediate SC are saccaderelated cells, some of which are directly related to the movement itself (so-called burst neurons), and others that show a long lead of low-frequency activation before a saccade (socalled buildup neurons), which are believed to be related to some form of saccade preparation (Dorris et al. 1997; Munoz and Wurtz 1995a). The fixation zone is thought to form the rostral end of the buildup layer, and the activity of fixation- and buildup-cells is believed to compete against one another in a push-pull relationship through lateral inhibitory connections (Munoz and Istvan 1998; Munoz and Wurtz 1995b). In addition, the level of buildup activity (i.e., discharge rate) is inversely related to saccadic latency (Dorris et al. 1997), and it has been suggested that collicular input from higher cortical areas (e.g., frontal eye fields: Segraves and Goldberg 1987; parietal cortex: Lynch et al. 1985) also influences this activity (Munoz and Wurtz 1995b), thereby affecting latency.

\footnotetext{
The costs of publication of this article were defrayed in part by the payment of page charges. The article must therefore be hereby marked "advertisement" in accordance with 18 U.S.C. Section 1734 solely to indicate this fact.
} 
A link between the fixation zone and the remote distractor effect was suggested by Walker et al. (1997). They argued that if fixation offset can decrease latency by decreasing activity in the fixation zone (allowing the early generation of a saccade), then visual onsets (i.e., distractors) within a certain central region might increase latency by increasing fixation cell activity at a critical time when a peripheral target appears. This possibility was implied earlier by Munoz and Wurtz (1993b) through a proposed link between the collicular fixation zone and the dead zone for express saccades (Weber et al. 1992). Munoz and Wurtz suggested that the onset of a stimulus within this region might also reactivate fixation cells, reducing the probability of express saccade generation.

In addition, Walker et al. (1997) suggested that the fixation zone may extend more peripherally than previously thought. They reported increased latencies with distractors presented in various spatial locations as much as 8 deg peripherally (although the greatest effect was for central distractors), with the exception of an approximately 20 deg target axis in which distractors instead modulated saccadic amplitude; that is, saccadic landing positions were between the target and distractor ("global effect": Deubel et al. 1984; Findlay 1982). Walker and colleagues therefore suggested that the fixation zone may extend as much as $8-10$ deg peripherally, and this effect has been similarly shown in terms of smooth pursuit latency (Knox and Bekkour 2004). Findlay and Walker (1999) recently incorporated this finding into their model of saccade generation and proposed that the effect is visually direct and not subject to cognitive influences.

There is also evidence that lateral interactions between competing subpopulations of active saccade-related collicular neurons can account for the remote distractor effect (Olivier et al. 1999; Trappenberg et al. 2001). Under this scheme, a saccade target and a remote distractor are believed to cause 2 separate locations of activation on the collicular motor map. Then through the lateral inhibitory network along the intermediate collicular layers (Munoz and Istvan 1998), the locus of target-related activity is delayed from reaching the level necessary for triggering a saccade.

There appears to be a growing consensus that virtually any form of visual stimulation near the time of target onset has a direct effect on a saccadic response, in particular saccadic latency, as evidenced by several studies (e.g., Baccino et al. 2001; Findlay and Walker 1999; Reingold and Stampe 2000, 2002; Vergilino-Perez and Findlay 2003; Walker et al. 1997; Weber and Fischer 1994). Most of these studies suggested the $\mathrm{SC}$ as a possible substrate behind their effect. For example, Reingold and Stampe (2000) reported an effect in which observers, during either a visual search task or a reading task, show an inhibited saccadic response (so-called saccadic inhibition) resulting from a short display change (e.g., a homogeneous blank field flashed for $33 \mathrm{~ms}$ ). They suggested 2 means through which collicular activity might account for this effect: 1) either the onset of the display stimulated competing subpopulations of buildup neurons, which through lateral inhibition might have delayed a saccadic response (Munoz and Istvan 1998); or 2) the display change stimulated an extended fixate system (Walker et al. 1997). Along similar lines, Baccino et al. (2001) reported that a bright background flicker (50 $\mathrm{Hz}$ ) starting at target onset and ending at saccade onset (i.e., during the latency period) increased saccadic latency to a peripheral laser point target (an effect particularly pertinent to concerns of CRT monitor use). They suggested that the background flicker acted like a display of remote distractors (in line with Walker et al. 1997) and they too implicate the SC as the neurological substrate behind the effect.

Although direct stimulation of neurons at the rostral pole of the SC stops saccades (Munoz and Wurtz 1993b), it is not clear whether visual events of any sort within the area representing the fixation zone should increase saccadic latency. In particular, it is not known whether these neurons discriminate between stimulation arising from spatially discrete elements (potential objects) and a background (a texture). Recall that Weber and Fischer (1994) reported an elimination of express saccades to a target in a large field of homogeneous distractors or simply with a distractor presented foveally on either side of fixation, presumably because the items stimulated the dead zone in either case. Weber and Fischer suggested that any display that affords pop-out might inhibit express saccades by control from the attention system allowing more efficient target detection. On the other hand, McPeek and Schiller (1994) reported that rhesus monkeys can make express saccades to a target in a display that affords pop-out, provided the number of distractors is very high, making the individual nontarget elements less salient as the display begins to form more of a texture. It appears then that not all forms of nontarget stimulation of the fixation zone result in increased saccadic latency. One possibility is that nontarget elements in larger displays may have to be clearly distinct before they are interpreted by the visual system as distractors.

The purpose of this research was to directly test whether nontarget stimulation of the region representing the collicular fixation zone is sufficient to produce an increase in saccadic latency, even if such stimulation is extended to cover a region beyond $2 \mathrm{deg}$ of the fovea. If this is the case, one might predict that a large form of distraction covering most of the visual field would increase saccadic latency as much as a small, foveally presented distractor (as suggested by Weber and Fischer 1994). However, if nontarget stimulation has to be a spatially confined element to produce a distractor effect, we may expect different results if we present a large spatially continuous nontarget (such as a texture). The research of McPeek and Schiller (1994) showed that nontarget stimuli must be visually discrete elements to suppress express saccades, and it may be implied that only in this case does the visual system interpret such stimuli as distractors. Thus a large texture should not increase latencies despite stimulation of the foveal fixation zone near the time of target onset. However, a reasonably small, foveally presented, patch of this texture might nonetheless be interpreted as a salient element. In this case, we predict that saccadic latency should increase.

In the present study, nontarget stimulation was in the form of a patch of pink noise (sometimes referred to as $1 / \mathrm{f}$ noise because it is a random noise texture whose power spectrum is approximately equal to 1 over the frequency). The power spectrum of pink noise is similar to natural images (which is about $1 / \mathrm{f}^{2}$; Burton and Moorhead 1987), making it an interesting setting for examining saccade target selection in its own right. The target was a stationary Gabor patch (4 cycles/deg, $\mathrm{SD}=0.7 \mathrm{deg}^{2}$ ), which always appeared 4,7 , or $10 \mathrm{deg}$ right or left of fixation. Sometimes the nontarget stimulus consisted of only a small central patch of noise, leaving the target visible 

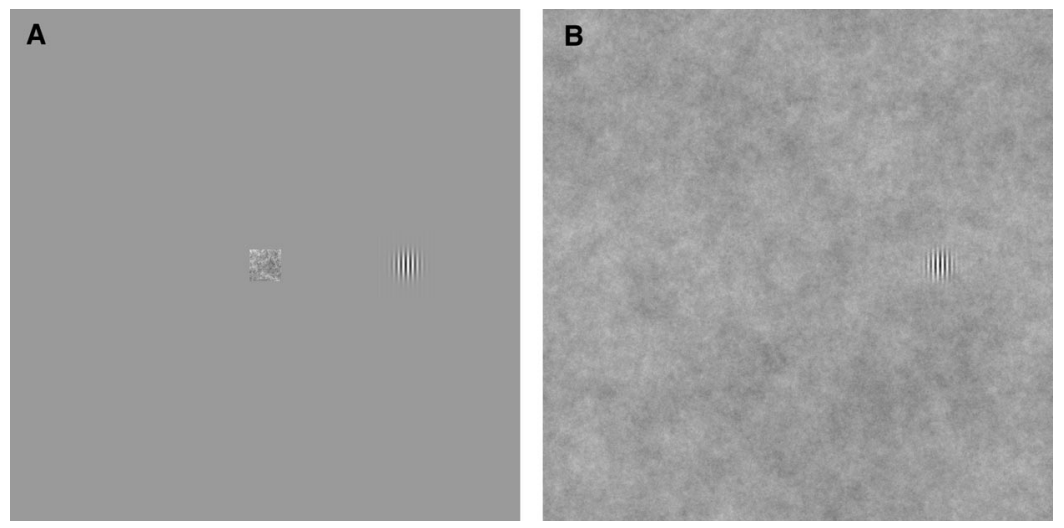

FIG. 1. A: sample of a small central patch of pink noise as a distractor, with a Gabor target approximately $10 \mathrm{deg}$ to the right. $B$ : sample of a $36 \times 36 \mathrm{deg}(1,024 \times 1,024$ pixels $)$ patch of noise with an embedded target at approximately 10 deg to the right. Both target and nontarget stimuli were presented at $50 \%$ contrast, with the same average luminance as the neutral gray background at $32 \mathrm{~cd} / \mathrm{m}^{2}$. Note that actual stimulus contrast and sizes were not exactly as shown here (for demonstration only). against a neutral gray background (see Fig. 1A). We compared this with a large patch of noise (essentially a background change), in which case the target was embedded in the noise (see Fig. $1 B$ ). We also examined the time course of these effects and the effect of varied patch sizes between these 2 extremes.

\section{METHODS}

\section{Observers}

Two authors plus 3 additional observers took part in each experiment. All observers had normal or corrected to normal visual acuity, and were experienced in psychophysical and saccade-related experimental tasks. Observers ranged from 20 to $35 \mathrm{yr}$ of age.

\section{Stimuli}

The target was a vertically oriented stationary Gabor patch (4 cycles/deg) with a Gaussian SD of $0.7 \mathrm{deg}^{2}$. The central fixation stimulus was a bull's-eye (i.e., black with a diameter of about $0.4 \mathrm{deg}$, and a neutral gray center of the same luminance as the background, 32 $\mathrm{cd} / \mathrm{m}^{2}$ ). Distractors consisted of a patch of pink noise, generated off-line using a MATLAB function (the size of the patches will be described in each experiment). Twenty-four randomly generated distractor patches were available for random selection on each trial of each experiment. Targets and distractors were presented at 50\% contrast and had the same average luminance as that of the neutral gray background on which they were displayed $\left(32 \mathrm{~cd} / \mathrm{m}^{2}\right)$. Previous testing revealed that contrast threshold of the target in the noise was slightly elevated relative to targets on the neutral gray background. ${ }^{1}$

\section{Equipment}

Stimuli were displayed on a 21-in. CRT monitor (ELO Touchsystems) driven by an ASUS V8170 GeForce 4 MX440 graphics board at a noninterlaced refresh rate of $100 \mathrm{~Hz}$. The resolution of the

\footnotetext{
${ }^{1}$ Thresholds to the stationary target Gabor in pink noise versus the neutral gray background were determined for observer BW using a staircase procedure. Both conditions with the various target eccentricities were randomly interleaved. Fixation was maintained with the aid of a central point, which remained visible throughout each trial. The target appeared randomly 4,7 , or $10 \mathrm{deg}$ left or right of fixation. After each trial the observer was prompted to respond on a game pad whether the target appeared left or right, and then at which eccentricity, "near," "medium," or "distant." Thresholds were calculated by taking the average contrast of a small sample of trials during the final reversals. This is similar to a suggested method by Macmillan and Creelman (1991) for staircase procedures, and provided reasonable thresholds for our purpose. Resulting thresholds for BW for targets 4, 7, and $10 \mathrm{deg}$, respectively, were $2.9,3.9$, and 5\% contrast against a neutral gray background, and 5.9, 7.7, and $7.2 \%$ contrast when the target was embedded in the noise. Target visibility was reduced in the noise.
}

monitor was set at $1,280 \times 1,024$ pixels, which corresponded to physical dimensions of $37 \mathrm{~cm}$ wide $\times 29.6 \mathrm{~cm}$ high. At a viewing distance of $47 \mathrm{~cm}$, the display occupied a retinal area of $45 \mathrm{deg}$ horizontally and 36 deg vertically. Eye movements were measured using EyeLink II (video-based tracker from SR Research, Mississauga, Ontario, Canada) at a sample rate of $250 \mathrm{~Hz}$.

\section{Procedure}

Calibrations were made before each block of trials (about every 90 to 140 trials), and consisted of fixating 9 consecutive bull's-eye stimuli at various locations on the screen. Average spatial accuracy for each calibration was maintained at $\leq 0.25 \mathrm{deg}$.

Figure 2 shows the sequence of a trial for each experiment. The fixation stimulus was present before the start of each trial. Observers had to fixate this stimulus and then initiate the trial, whenever they were ready, by pressing a key on a game pad. This allowed for a drift correction procedure at the start of each trial. In the event that observers were not fixating accurately (within $1 \mathrm{deg}$ ), an error tone was presented and the trial had to be reinitiated. In all experiments, the fixation was extinguished $1,000 \mathrm{~ms}$ after the start of a trial, and the patch of pink noise, when present, was onset at the same time in the same central location (with the exception of expt. 1 in which the distractor appeared centrally, or 4, 7, or $10 \mathrm{deg}$ above or below the fixation location). A control condition in which no distractor appeared was also always used. With the exception of expt. 3, the target also appeared simultaneously with these events, 4, 7, or 10 deg randomly left or right of fixation, and remained present for 1,000 ms. In expt. 3 we varied the onset of the target $(-100,-50,0,50$, or $100 \mathrm{~ms})$ relative to fixation offset and noise patch onset. The result was a $3 \times$ 5 factorial design [ 1 control and 2 distractor conditions $\times 5$ target stimulus onset asynchronies (SOAs)].

Observers were simply requested to make an eye movement to the center of the target when it appeared, and that both speed and accuracy

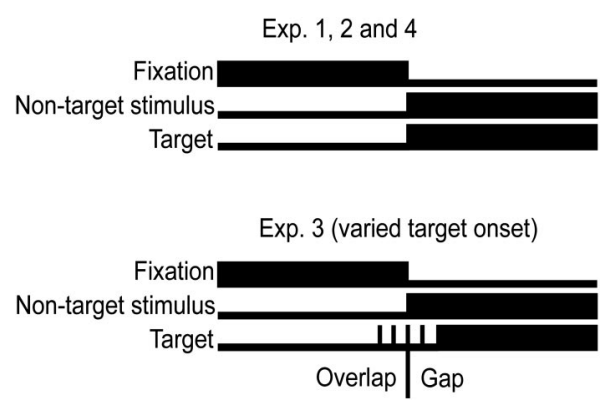

FIG. 2. Sequence of events on a given trial. Except for expt. 3 (time course of target onset), all events occurred simultaneously (1,000 ms after trial initiation). In expt. 3, target onset was varied relative to the remaining events. Thus when the nontarget stimulus was absent, the result was a fixation gap/overlap paradigm, which served as the control condition. 
are equally important. The observer's head was stabilized by a chin rest. Each observer completed $\geq 18$ trials per condition of each experiment. Eye position and event data were recorded, and all analyses were done off-line.

\section{Analyses}

Trials with direction errors, or eye traces that drifted more than 1 deg vertically or horizontally from fixation before a saccade, or more than 2 deg vertically during a saccade, were removed from analyses. In terms of accuracy, we calculated both signed and unsigned error of individual trials. Signed error was the distance of a saccade landing position from the target (in deg) along the horizontal axis, such that undershoots were represented as negative values and overshoots were represented as positive values. Unsigned error was the absolute value of the distance of the saccade landing positions from the target (in deg). Latency was the time between target onset and the onset of the first saccade, which was based on a velocity criterion $\geq 30 \mathrm{deg} / \mathrm{s}$. No outlier procedure was used, but we removed trials with an error $>3$ $\mathrm{deg}$, as well as trials with latencies $>600$ or $<80 \mathrm{~ms}$ (saccades $<80$ ms are presumably anticipatory; Wenban-Smith and Findlay 1991). With these criteria, $<5 \%$ of the total number of trials was removed, indicating that the target was clearly visible, and that the task was reasonably easy regardless of distractors.

We derived each subject's median latency, and median signed- and unsigned error, for each condition. It should be noted that all figures report the mean of the subjects' median values. With the exception of expt. 1, the data were collapsed across target positions (i.e., eccentricities and left vs. right locations). We used the various target eccentricities only for the purpose of decreasing predictability of the target's location.

\section{Experiment 1}

The purpose of expt. 1 was to demonstrate that the distractor effect might be obtained with a small patch $(64 \times 64$ pixels, $2.3 \times 2.3 \mathrm{deg})$ of pink noise as a distractor stimulus. When present, the distractor patch appeared either foveally, or 4, 7, or 10 deg above or below fixation along the vertical meridian, while the target appeared simultaneously along the horizontal meridian (randomly at one of the previously mentioned target locations). Walker et al. (1997) previously showed distractor effects for nontarget stimuli in various spatial locations (including the vertical axis), with the strongest effect for foveally presented distractors. We predicted a steep effect of eccentricity as well, with the strongest effect for the central distractor.

RESULTS AND DISCUSSION. Accuracy. Figure 3, $A$ and $B$ show mean signed and unsigned error, respectively, as a function of distractor eccentricity. There appeared to be more undershooting in the presence of peripheral distractors (Fig. $3 A$, signed error), so we ran a repeatedmeasures ANOVA on the 5 conditions represented in the graph (i.e., 1 no-distractor control and 4 distractor eccentricities). However, the test was not significant $[F(4,16)=1.72, P=0.19]$, indicating that the distractors did not significantly affect saccadic accuracy. Presumably distractors outside approximately $20 \mathrm{deg}$ of the target axis have no effect on accuracy (Walker et al. 1997). Our data are consistent with this.

Latency. Figure $3 C$ shows the mean saccadic latency as a function of distractor eccentricity for each target eccentricity separately (thin lines) and for the combined target eccentricities (solid, thick line). Presumably the remote distractor effect is also influenced by the ratio of distractor-to-target eccentricity (Walker et al. 1997). This does not appear to be the case in our data (Fig. $3 C$, thin lines). The latency pattern looks nearly the same regardless of target eccentricity. If such an effect is present in our data, we should see a significant interaction between target and distractor eccentricity, so we ran a 3 (target eccentricity) $\times 4$ (distractor eccentricity) repeated-measures ANOVA to test whether this was in fact the case. The effect of target eccentricity was not significant $[F(2,24)<1]$ and, more important, the interaction between target and distractor eccentricity was also not significant $[F(6,24)=1.33, P=0.28]$. This indicates that any effect of the distractors did not vary as a function of target eccentricity,
A

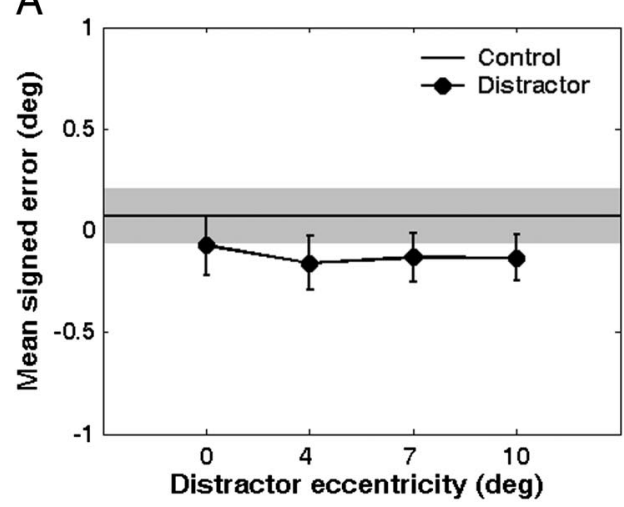

C

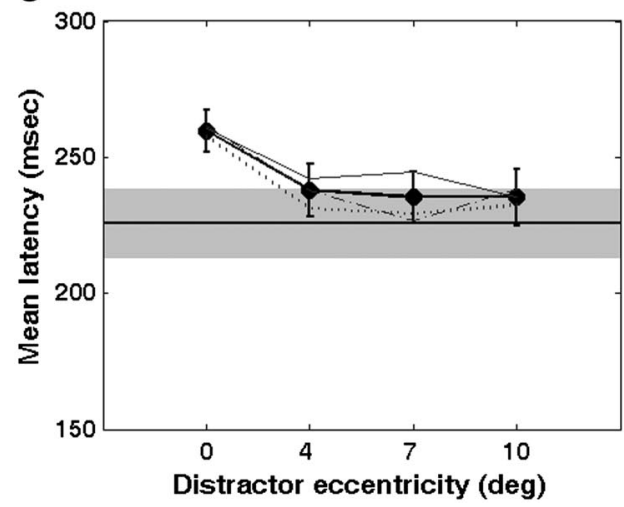

B

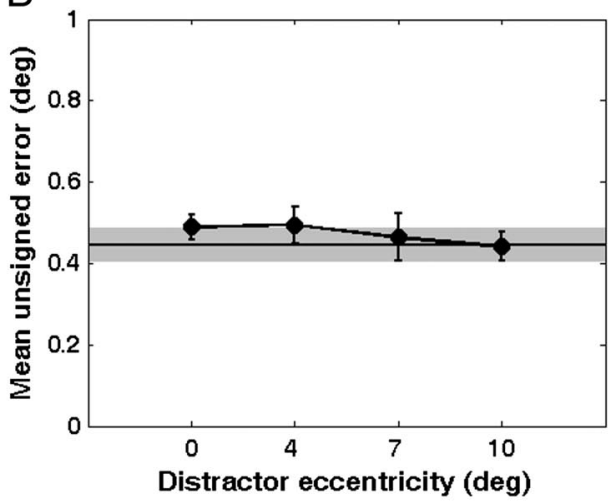

D

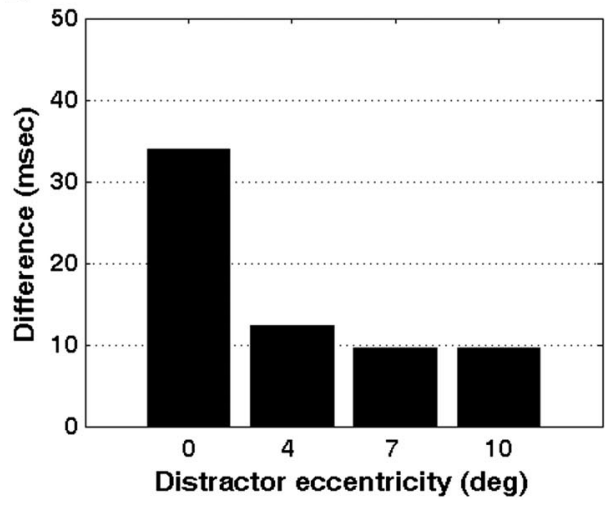

FIG. 3. Mean signed error $(A)$ and unsigned error $(B)$ along the target axis as a function of distractor eccentricity. $C$ : thick, solid line represents mean saccade latency as a function of distractor eccentricity (target eccentricities combined). Error bars represent 1 SE. SE for the control condition (solid line) is represented by the shaded gray area. Three thin lines represent mean saccade latency for each target eccentricity separately; the 4, 7, and 10 deg targets are represented by the thin dotted, dashed, and solid line, respectively. $D$ : difference values represent the mean latency of each distractor condition minus the mean latency of the control condition (target eccentricities combined). 
although the effect of distractor eccentricity was significant $[F(3,24)=14.19, P<0.001]$. This can be clearly seen in Fig. $3 D$, which shows the difference between the distractor conditions minus the control condition (combined target eccentricities). There appeared to be a clear increase in latency for the 0 deg distractor condition relative to the control condition, but there was no obvious difference across the peripheral distractor conditions $(>0 \mathrm{deg})$, so we collapsed these into one mean, and then ran 2 Bonferroni-adjusted pairedsamples $t$-test ( 0 deg distractor with the control, and peripheral distractors with the control). Only the central distractor showed a significant increase in saccadic latency relative to the control condition $[t(4)=$ 3.66, $P=0.02]$. Latency for the combined peripheral distractors was not significantly different from the no-distractor control condition $[t(4)=2.77, P=0.08]$. In short, a small patch of centrally displayed pink noise increased saccadic latency by about $34 \mathrm{~ms}$.

The lack of a significant effect for more peripheral distractors may be inconsistent with the idea of an extended fixation zone (Walker et al. 1997). However, part of the support for the idea comes from the fact that the magnitude of the effect is modulated by the distance of the distractor from the fovea, as well as physiological evidence that the proportion of direct projections from the SC to omnipause neurons in the brain stem (the activity of which is directly related to saccade initiation and termination) decreases gradually as we move away from the rostral pole (Gandhi and Keller 1997). Although our effect for peripheral distractors was not significant, it is reasonably within the range of latencies reported by Walker et al. (1997) with similar target and distractor positions (i.e., about 10 to $20 \mathrm{~ms}$ ). Nonetheless, our effect appears to drop dramatically between the central and peripheral distractors, and then remains level up to a distractor eccentricity of 10 deg. This might suggest the involvement of separate mechanisms, one involving the fixation system (although not extending to the point proposed by Walker et al. 1997) and the other involving inhibitory lateral interactions along the remainder of the collicular motor map (Olivier et al. 1999).

We agree with Tam (1999) that an increase in fixation activity by a peripheral stimulus appears counterintuitive. Evidence against an extended fixation zone has also been suggested by the work of Fendrich et al. (1999). They used a gap/overlap paradigm in which the fixation stimulus was a square formed by 4 peripheral anchor points appearing 1,2, or 4 deg from the square's center (the target appeared $6 \mathrm{deg}$ left or right of center). These authors found that saccadic latency was facilitated (between a gap 0 and continuous overlap condition) only with fixation anchors $<2 \mathrm{deg}$, which is inconsistent with the idea of an extended fixation system.

\section{Experiment 2}

Once we established a significant distractor effect using a small patch of pink noise, we then compared this to the onset of a patch covering a larger area of the visual field $(36 \times 36 \mathrm{deg}$, essentially a background change). In this case the target was embedded in the noise (Fig. $1 B$ ). Previous testing revealed that the visibility of the target is slightly reduced in the noise relative to a neutral gray background (see footnote 1 above). Recall that Weber and Fischer (1994) used a similar comparison using oriented lines as stimuli (large vs. small central display) and found no differences between the conditions (in terms of the proportion of express saccades ${ }^{2}$ ). Given this fact, and the assumption that visual stimulation of an extended fixation zone can account for the effect of remote distractors (Walker et al. 1997), one

\footnotetext{
${ }^{2}$ We recognize the distinction between comparing proportions of express saccades with mean latencies. With the exception of expt. 3, we did not use a gap paradigm. As such, there was no indication of bimodality in our data, so we focused on mean latencies instead of proportions of express saccades. Nonetheless, variations in proportions of express saccades would also likely influence average latencies in a consistent manner, and both dependent measures have been shown to be reasonable indicators of a distractor effect, which was the primary issue.
}

might predict that latencies would increase in either the small or large patch condition. However, if nontarget stimulation has to appear as discrete elements to be interpreted as a distractor, as suggested by the research of McPeek and Schiller (1994), the large pink noise texture used here should not increase saccadic latency.

RESULTS AND DISCUSSION. Accuracy. Figure 4, $A$ and $B$ show mean signed and unsigned error, respectively, along the target axis for each condition. There is clearly no difference between either of the conditions. Accuracies were virtually identical regardless of the presence of nontarget stimulation.

Latency. Figure $4 C$ shows mean latency for each of the conditions, and Fig. $4 D$ shows the size of the effects. There was a significant effect of condition $[F(2,8)=12.98, P<0.005]$. Subsequent Bonferroni-adjusted $t$-test revealed that only the small patch significantly increased latency $[t(4)=3.92, P=0.017]$.

As predicted, nontarget stimulation of the region representing the fixation zone may be necessary, although it is not in itself sufficient to produce the increase in latency typically found with remote distractors. Moreover, we know visibility of the target embedded in the noise was reduced relative to a neutral gray background (see footnote 1 above). We made no attempt to control for this so as to make the test more conservative.

\section{Experiment 3}

Experiment 3 was designed to examine the temporal dynamics of the effects found in expt. 2 . We used both the small $(2.3 \times 2.3 \mathrm{deg})$ and the large $(36 \times 36 \mathrm{deg})$ nontarget stimuli, the onset of which remained simultaneous with fixation removal. The only thing that changed was that the target could appear at various onset asynchronies $\leq 100 \mathrm{~ms}$ before or after the distractor and fixation events (Fig. 2). Thus in the absence of a nontarget stimulus (i.e., the control condition), target SOAs $>0$ resulted in a gap condition, and target SOAs $<0$ resulted in an overlap condition.

With this design, it could be argued that the onset of a central nontarget stimulus (in particular, the small patch of pink noise) simultaneous with fixation offset acts as an extended fixation event. However, the switch from a fixation stimulus to a distractor is perhaps better defined as a change event (Ross and Ross 1980). This change remains central to our study's primary purpose: that is, whether a switch from a fixation stimulus to another form of raw visual stimulation necessarily inhibits a saccadic response (presumably by sustaining the tonic firing rate of fixation neurons). In addition, a change or an onset at fixation has been shown to produce the same increase in saccadic latency (Ross and Ross 1980; see Walker et al. 1997, who noted this as well). Thus this experiment should allow us to test how the effect of each change event (i.e., nontarget stimulus) might vary with target SOA, in comparison to the no-change control condition. The control condition is expected to have its own effect of SOA as we move from fixation gap to overlap, and the question is what additional effect will the simultaneous change event have, if any?

Ross and Ross (1980) showed that a change at fixation before the onset of a saccade target can act as a warning signal, provided the interval is $>100 \mathrm{~ms}$. When the change- and target event were simultaneous, the result was a latency increase consistent with the central distractor effect reported by Walker et al. (1997) and our current study. Braun and Breitmeyer (1990) showed that the reappearance of a fixation stimulus $100 \mathrm{~ms}$ or more before target onset can also facilitate saccade latency relative to its reappearance simultaneously with the target. More recently, Walker et al. (1995) examined the time-course effect of a distractor appearing in left hemifield while the target appeared mirror opposite this in the right hemifield (the fixation point remained continuously present). Again, distractors presented close to target onset (and slightly thereafter) produced the greatest latency increase, but if presented more than $100 \mathrm{~ms}$ before the target, latencies were facilitated. 
A

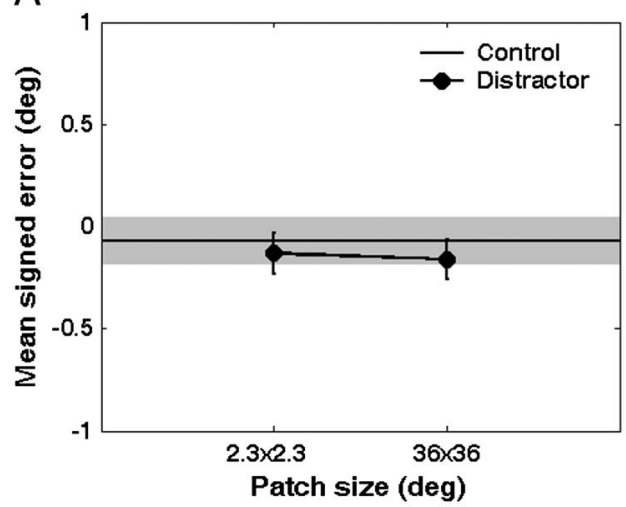

C

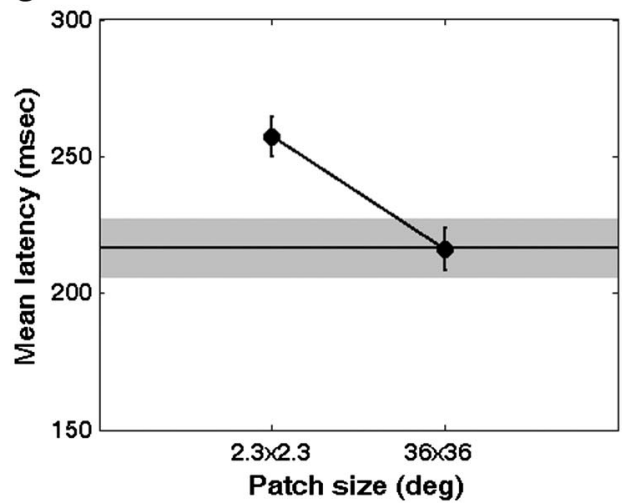

B

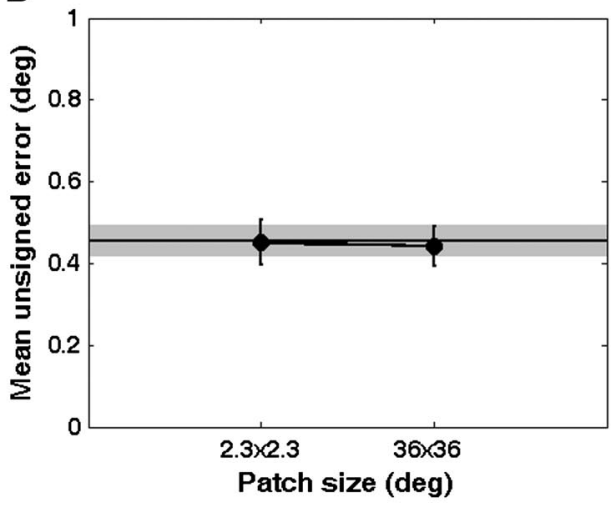

D

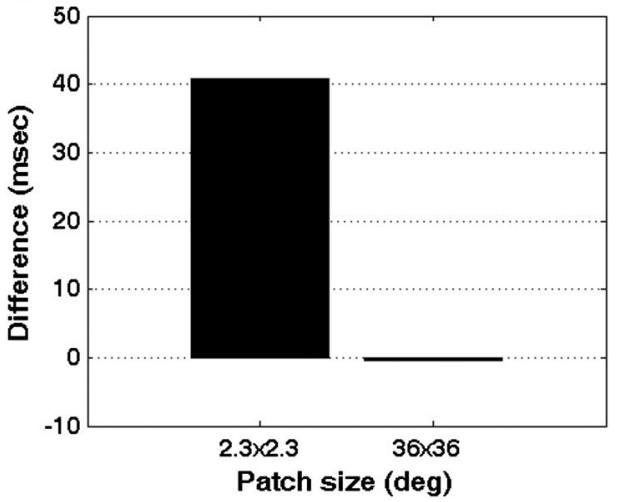

FIG. 4. Mean signed error $(A)$ and unsigned error $(B)$ along the target axis as a function of pink noise patch size. $C$ : mean latency as a function of patch size. Error bars represent 1 SE. SE for the control condition (solid line) is represented by the shaded gray area. $D$ : difference values represent the mean latency of the noise patch condition minus the mean latency of the control condition.
The onset of a nontarget stimulus then can facilitate saccadic latency by acting as a warning signal, although it appears to require at least a 100-ms distractor-to-target lead. It is unlikely then that the maximum distractor-to-target interval used in our paradigm (100 ms) will result in a significant warning effect with the small centrally displayed patch of pink noise. However, because the large patch of noise was not interpreted as a distractor in expt. 2, it might provide a reasonable warning signal. In addition, as the change event begins to occur after target onset (at target SOAs $<0$ ), we were interested in whether the large patch change event would begin to interfere with the saccade.

RESUlTS AND DISCUSSION. Accuracy. Figure 5, $A$ and $B$ show the mean signed and unsigned error, respectively, along the target axis for each condition. As with the previous 2 experiments, there were no obvious differences between conditions in terms of accuracy, except for a possible increase in saccadic undershooting, and increased unsigned error, in the large noise patch condition as target SOA increased $>0 \mathrm{~ms}$. We ran a repeated-measures ANOVA on the mean unsigned error for the large patch condition only, across target SOAs. The test was significant $[F(4,16)=5.85, P<0.01]$, suggesting at least some decrease in accuracy to targets in the background of pink noise as the gap between patch onset and target onset increased (although post hoc tests were not significant). It could be argued that some local regions of the large texture of pink noise stimulated neighboring locations to the target causing a "global effect" (Deubel et al. 1984; Findlay 1982). However, the location of such a region should be random and might just as likely cause a latency increase similar to that of typical remote distractors (Walker et al. 1997), which clearly did not happen.

Latency. Figure $5 C$ shows mean latencies for each condition across target SOAs. In terms of the control condition we see the expected decrease in latency as target SOA increases from a -100 -ms fixation overlap to a +100 -ms gap. If we consider the distractor conditions relative to this, we also see a decline in latency as SOA increases, but there is also a noticeable interaction: Latencies in the small patch condition remain elevated relative to the control between SOAs of -100 and $0 \mathrm{~ms}$, whereas there is virtually no difference between the large patch condition and the control up to this point. At target SOAs greater than this the pattern begins to turn around, as latencies in the large patch condition continue to decrease, whereas differences between the small patch condition and the control become smaller.

Figure $5 D$ shows the difference between control and distractor conditions across target SOA. Scores in the positive direction indicate a facilitatory effect on latency (i.e., a latency decrease), and negative scores indicate an increase in latency. Given the similarity in latency difference scores between SOA of -100 to $0 \mathrm{~ms}$, and between +50 and $+100 \mathrm{~ms}$, we collapsed the data into 2 temporal categories (SOAs $\leqq 0$, and SOAs $>0$ ) before running tests. We then ran a $2 \times 3$ (i.e., 2 target SOAs $\times 3$ stimulus conditions) repeated-measures ANOVA on the resulting mean latencies. There was a significant interaction between SOA and stimulus conditions $[F(2,8)=34.17, P<0.001]$.

We were primarily interested in differences of nontarget stimulus conditions relative to the no-distractor control condition, so we ran only 4 Bonferroni-adjusted paired-samples $t$-test to tease apart the interaction: the small patch versus control, and the large patch versus control, both at the early temporal period (SOAs $\leqq 0$ ), and at the later temporal period (SOAs $>0$ ). Table 1 shows the mean latencies and difference scores for these conditions. In short, only the small patch showed an effect at the early temporal period, and only the large patch showed an effect at the later temporal period. That is, the small central patch of pink noise resulted in a significant distractor effect, but only at target SOAs of $\leqq 0$. The large patch of pink noise actually reduced latencies relative to the control condition when it was onset before the target. This is surprising for a number of reasons: First, the effect of the large patch of pink noise (at SOAs $\geqq+50$ ) decreased latency relative to the effect of fixation gap, which is known to reduce latency itself. In addition, the small patch produced no such facilitatory effect, despite the fact that it was centrally displayed and clearly visible, as 
A

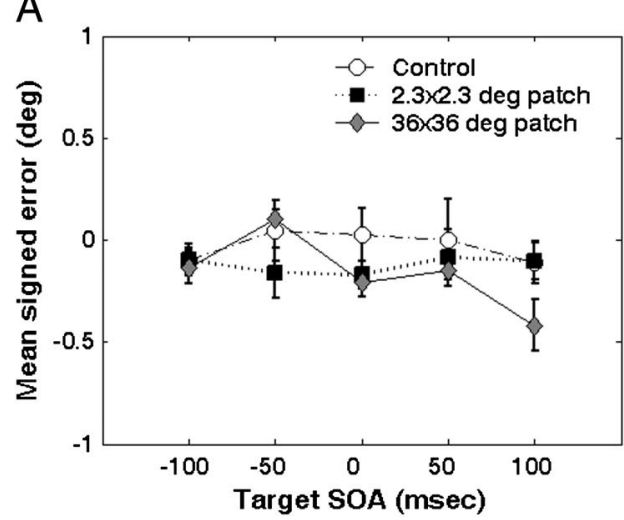

C

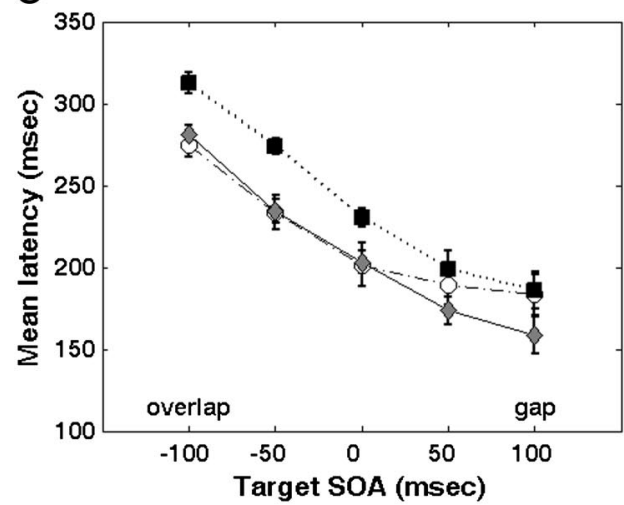

B

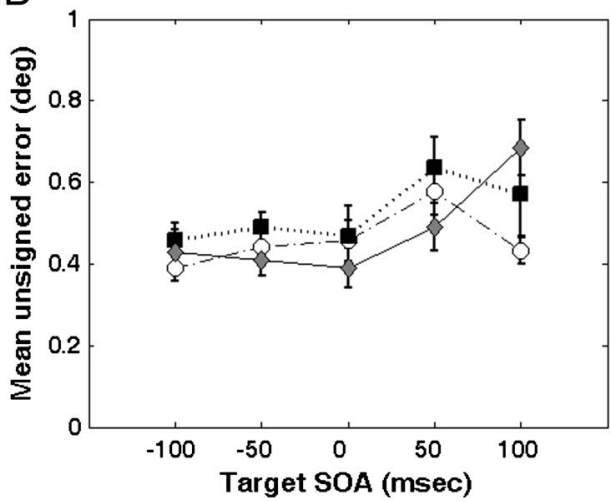

D

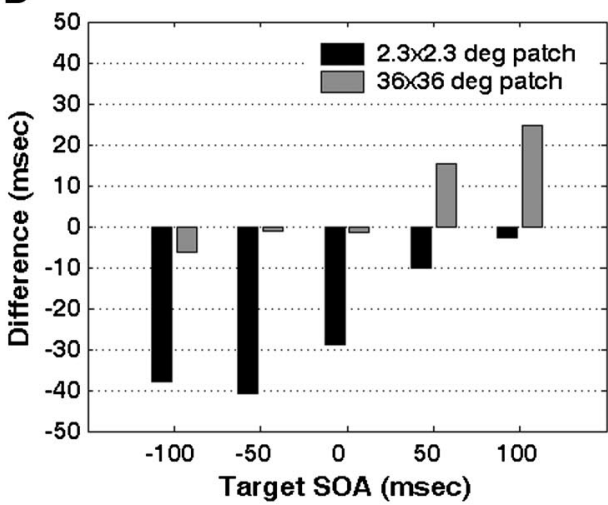

FIG. 5. Mean signed error $(A)$ and mean unsigned error $(B)$ along the target axis as a function of distractor condition and target stimulus onset asynchrony (SOA). $C$ : mean latency as a function of distractor condition and target SOA. Error bars represent 1 SE. $D$ : difference values represent the mean latency of the control condition minus the mean latency of the distractor condition for each target SOA. Positive scores represent a facilitatory effect (i.e., latency decrease) relative to gap (target SOAs $>0$ ) and overlap (target SOAs $<0$ ) control conditions. Negative scores represent increased latency with respect to the control conditions. evidenced by the significant latency increase at SOAs $\leqq 0$. Furthermore, recall that the visibility of our target was reduced in the background of noise, relative to neutral gray (see footnote 1 above). This effect is also in strong contrast to research suggesting a display change causes in an inhibited saccadic response (Reingold and Stampe 2000, 2002).

Facilitation from the large patch may be at least partially a warning effect. Distractor stimuli have been shown to produce warning effects, but typically at earlier onsets relative to the target (gaps of $>100 \mathrm{~ms}$; Braun and Breitmeyer 1990; Ross and Ross 1980; Walker et al. 1995). It is possible that because the large noise display was not interpreted as a distractor, its effect as a warning signal may have been more effective than the small patch. Nonetheless, at least part of the facilitatory effect of fixation gap is attributed to a warning signal (Kingstone and Klein 1993; Reuter-Lorenz et al. 1995), and the prior onset of the large patch of random noise in this experiment significantly reduced latency relative to this. We will discuss another possibility later related to the statistical property of pink noise.

We recognize that there is some indication of a leveling-off of the gap effect in the control condition from an SOA of 0 to +100 (Fig. $5 C$ ). It could be argued that the interaction is attributed to this rather

TABLE 1. Mean latencies and difference scores for nontarget stimulus conditions relative to no-distractor control condition as a function of SOA (Expt. 3)

\begin{tabular}{lll}
\hline \hline \multicolumn{1}{c}{ Condition } & \multicolumn{1}{c}{ SOA $\leqq 0$} & \multicolumn{1}{c}{ SOA $>0$} \\
\hline No-distractor control & $M=236, \mathrm{SE}=9.9$ & $M=186, \mathrm{SE}=12.2$ \\
$2.3 \times 2.3$ deg patch & $M=272, \mathrm{SE}=5.3$ & $M=192, \mathrm{SE}=11.3$ \\
$36 \times 36$ deg patch & $M=239, \mathrm{SE}=6.6$ & $M=166, \mathrm{SE}=9.8$ \\
Control—small patch & $M$ diff $=-36, * \mathrm{SE}=4.9$ & $M$ diff $=-6, \mathrm{SE}=3.1$ \\
Control-large patch & $M$ diff $=3, \mathrm{SE}=4.1$ & $M$ diff $=20, * \mathrm{SE}=4.1$ \\
\hline
\end{tabular}

$* P<0.01$ (with Bonferroni adjustment). than the nontarget stimulus conditions. However, despite the robustness of the gap effect, its size is likely to depend on the degree to which the conditions under which it is tested facilitate express saccades (Wenban-Smith and Findlay 1991). We believe that the reduced target predictability in our study compared to that of typical gap studies explains part of this discrepancy (Schiller et al. 2004). Had express saccades been present in our data, we suspect that the slope of both the control and nontarget stimulus conditions would have been steeper during the gap interval, but the interaction would still be present. Furthermore, all trials from all conditions were randomly interleaved, so we see no systematic reason why the interaction might be more attributable to the control rather than to experimental conditions. It is also important to note that there is consistently no deviation between the control and large patch condition up to an $\mathrm{SOA}=0 \mathrm{~ms}$ (Fig. $5 C$ ). This provides a clear indication that the large patch of noise had no effect on the mechanisms influencing saccade latency during this period. Only after an SOA $>0$ do we begin to see a difference, which can be attributable only to the single difference between these two conditions: the onset of the large nontarget stimulus.

\section{Experiment 4}

We have established that a small $(2.3 \times 2.3 \mathrm{deg})$ patch of pink noise can result in a significant distractor effect even if presented $\leq 100 \mathrm{~ms}$ after target onset, and that the opposite effect can occur with prior onset of a larger portion $(36 \times 36 \mathrm{deg})$ of the same stimulus. The purpose of this experiment was to test various patch sizes between these 2 extremes. One limitation was that we could increase the patch of noise only up to a certain size, after which it would overlap with the least eccentric target location. Thus only patch sizes below this limit were used. Nonetheless we were able to vary the size from just below the limit of the collicular fixation zone, as defined by Munoz and Wurtz (1993a), to reasonably above it. Each unit of size increase was nearly double the area of the former. Physical patch sizes were $45 \times$ 
45 pixels, $64 \times 64$ pixels, $90 \times 90$ pixels, and $128 \times 128$ pixels. This corresponded to visible patch sizes of about $1.6 \times 1.6 \mathrm{deg}, 2.3 \times 2.3$ $\mathrm{deg}, 3.2 \times 3.2 \mathrm{deg}$, and $4.5 \times 4.5 \mathrm{deg}$, respectively.

We were unsure whether we would see an initial increasing distractor effect as the size of the noise patch increased, or if the effect would begin to fade immediately toward that which we found for the large portion of noise. Weber and Fischer (1994) found an increasing effect as the size of a small, square distractor increased from $0.1 \times 0.1$ deg to $0.2 \times 0.2 \mathrm{deg}$ to $0.4 \times 0.4 \mathrm{deg}$ (the target being also a small square of $0.2 \times 0.2 \mathrm{deg}$ ). Although these stimuli were much smaller than ours, Weber and Fischer's data do indicate that the effect is particularly sensitive to small changes in size.

Because the effect of remote distractors decreases with eccentricity (Walker et al. 1997), it could be argued that an effect of distractor size in this experiment is the result of the high-contrast edges stimulating increasingly eccentric locations along the presumed fixation zone, rather than the surface area of the patch itself. Thus we included additional data from 4 of our subjects on a control experiment using edge-filtered patches. These patches were generated by gradually, eccentrically reducing contrast at the edges to $0 \%$ at a rate of about $3.3 \%$ per pixel over 30 pixels (about $1 \mathrm{deg}$ ). Visibly, the edge-filtered patches were designed to be nearly identical in size to the originals. The data from this experiment are included within the same plots.

RESUlts AND DISCUSSION. Accuracy. Figure $6, A$ and $B$ show the mean signed and unsigned error, respectively, along the target axis for each condition. The gray circles with dotted lines represent the data of the 4 subjects that also completed the edge-filtered control experiment. What is immediately obvious is the overall greater amount of overshooting (signed error, Fig. 6A) in the edge-filtered experiment. Because this was also true between the no-distractor control conditions of the original and edge-filtered experiment (which were essentially identical in design), we attribute the difference to factors outside our experimental manipulation. More important, there were no significant differences in accuracy between distractor and no-distractor condi- tions for either the original experiment [signed error, $F(4,16)=1.70$, $P=0.20$; unsigned error, $F(4,16)=1.44, P=0.27]$ or the edgefiltered experiment [signed error, $F(4,12)=1.46, P=0.54$; unsigned error, $F(4,12)=1.02, P=0.43]$. Thus saccadic accuracy was again not significantly affected by the presence of our nontarget stimuli.

Latency. Figure $6 C$ shows the mean latency for each of the conditions. Figure $6 D$ shows the size of the effect. The edge-filtered control experiment is represented by the open circles with the dotted line. As can be seen, latencies showed a steady decrease as the size of the central pink noise distractor increased, and the same is true for the edge-filtered control experiment. A repeated-measures ANOVA revealed a significant effect of patch size $[F(3,12)=5.31, P<0.05$ (original experiment), and $F(3,9)=8.15, P<0.01$ (edge-filtered experiment)].

The results of expt. 4 give us a sense of the degree to which the distractor effect is modified by changes in distractor size. Because the same result was obtained in the edge-filtered control experiment, it is unlikely that the effect arose from the high-contrast edges stimulating increasingly eccentric locations of an extended fixation zone. The effect is therefore more likely related to the overall surface area of the patches. One possibility is that modification of the nontarget size is related to the degree to which it is interpreted by the visual system as a salient element in the visual field. Whether this is based on some absolute size or relative to the target size would require further testing. Physiologically, this effect might also stem from the possibility that the smaller patch is simply better at driving fixation mechanisms. Nonetheless, this experiment again brings into question the proposed nature of the mechanisms underlying increased saccadic latency ascribed to the appearance of nontarget stimuli.

\section{GENERAL DISCUSSION}

In sum, the experiments presented here supported our predictions: We demonstrated that the remote distractor effect is
A

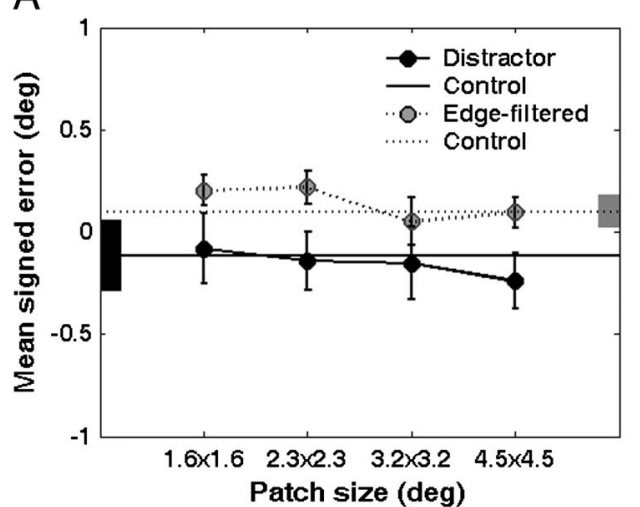

C

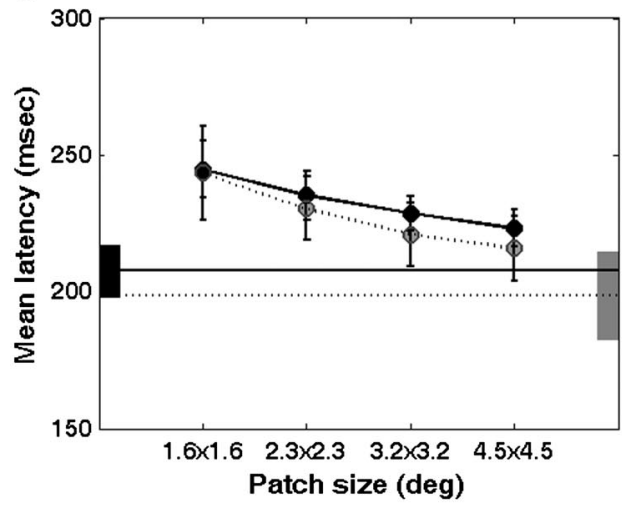

$\mathrm{B}$

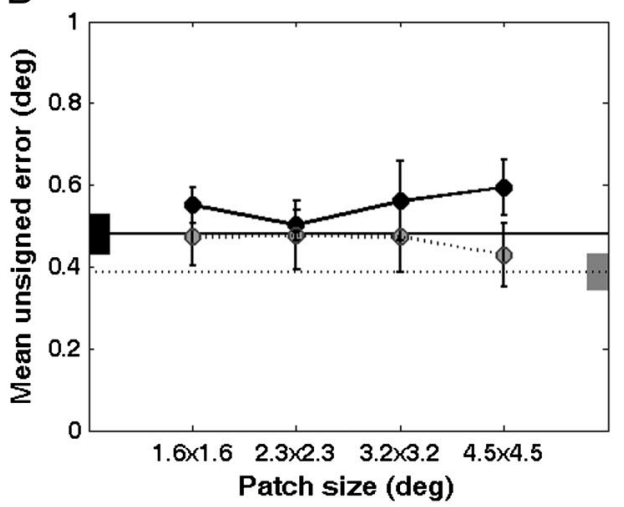

D

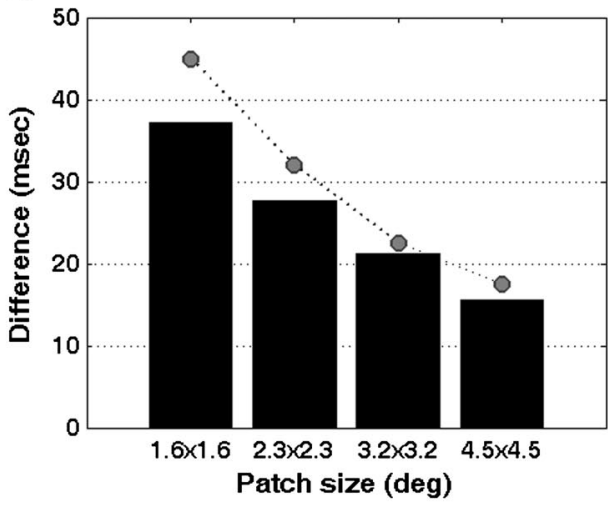

FIG. 6. Mean signed error $(A)$ and unsigned error $(B)$ along the target axis as a function of size of central distractor patch. Gray circles with dotted lines represent the edge-filtered control experiment. No-distractor control condition of this experiment is represented by the flat dotted line. $C$ : mean latency as a function of the size of central distractor. Error bars represent 1 SE. SE for the no-distractor control conditions is represented by the shaded black region on the left (primary experiment), and the shaded gray region on the right (edge-filtered experiment). $D$ : difference values represent the mean latency of the noise patch condition minus the mean latency of the control condition. Note that each increase in patch size represents approximately twice the area of the former (e.g., $1.6^{2}, 2.3^{2}, 3.2^{2}$, and $4.5^{2}=$ 2.6, 5.3, 10.2, and 20.3, respectively). 
possible with a small patch of pink noise as a distractor (expt. 1). More important, we confirmed that raw visual stimulation of the central region representing the collicular fixation zone is not in itself sufficient to produce a significant increase in saccadic latency to a simultaneously presented target (expt. 2). This may have been implied from the study by McPeek and Schiller (1994), although to the best of our knowledge no one has made this distinction or put it to a direct test. Recall that McPeek and Schiller showed that the numerosity of nontarget elements in a pop-out display affects whether express saccades are possible (i.e., only a background of very densely packed nontarget stimuli permitted express saccades). Presumably densely packed nontarget stimuli permit rapid oculomotor target selection because there are no distinct elements that the visual system might interpret as a distractor. It is possible that the large $(36 \times 36 \mathrm{deg})$ nontarget display of pink noise in the present study did not increase latency for the same reason (see footnote 2 above).

Physiologically, it is possible that the display-sized nontarget stimulus used here was simply not adequate to drive the cells responsible for visual fixation. It is also unlikely that local regions of this random noise texture activated other subpopulations of saccade-related collicular neurons, which might have subsequently increased latency through lateral inhibitory interactions (Munoz and Istvan 1998; Olivier et al. 1999). It is questionable then whether a background change without the presence of obviously salient elements should drive fixation- or saccade-related collicular cells. Recall that Reingold and Stampe (2000, 2002) reported an inhibited saccadic response as a result of a flashed display change (e.g., a homogenous blank field). This inhibitory effect was shown to occur as early as $60 \mathrm{~ms}$ from the onset of the flashed event, so Reingold and Stampe implicated the SC as the only possible substrate behind the effect because it can receive direct visual input in as little as $35 \mathrm{~ms}$ (Rizzolatti et al. 1980). In essence, they argue that either the onset of the display stimulated competing subpopulations of buildup neurons, which through lateral inhibition might have delayed a saccadic response (Munoz and Istvan 1998), or the display change stimulated an extended fixate system (Walker et al. 1997). Similarly, Baccino et al. (2001) interpreted the effect of a background flicker on saccadic latency as "an area of remote distractors that inhibit, i.e., produce longer latencies in simple horizontal saccades," in reference to Walker et al. (1997).

What then might account for the difference between our data and the saccadic inhibition effect reported by Reingold and Stampe? Unfortunately it is not possible to adequately answer this question without further research. We know, however, that the difference is not likely attributable to a luminance change between the original and change display. Both target and nontarget stimuli in our experiments had the same average luminance $\left(32 \mathrm{~cd} / \mathrm{m}^{2}\right)$, and the effect reported by Reingold and Stampe (2000) has been shown with an original and change display of equal luminance. Although the change in Reingold and Stampe's paradigm is flashed for only $33 \mathrm{~ms}$ (whereas ours remained present once onset), we suspect that this also cannot account for the difference.

In addition, we have shown that the prior onset of the display-sized pink noise texture (expt. 3) can reduce latencies relative to a neutral gray control condition, and this effect occurs at a shorter nontarget to target gap ( $\leqq 100 \mathrm{~ms})$ than is typically found with distractors as warning signals $(>100 \mathrm{~ms}$; Braun and Breitmeyer 1990; Ross and Ross 1980; Walker et al. 1995). We suggested that the onset of this large texture might have provided a more effective warning signal because it was not simultaneously interpreted as a distractor like the small patch. Nonetheless, at least part of the effect of fixation offset is assumed to be attributed to a warning signal (Kingstone and Klein 1993; Reuter-Lorenz et al. 1995), and mean latency in our large noise-patch condition was significantly reduced relative to this.

Another possibility alluded to earlier is that the structure of a pink noise texture has some special properties that might permit rapid saccade target selection. For example, there is evidence that the visual system is optimized for the 2nd-order (i.e., Fourier) composition of natural images in general (Párraga et al. 2000) and for detecting animals in such images (Thorpe et al. 2001). Saccadic target selection may also be optimized for the 2nd-order image properties of pink noise given its similarity to natural images in this regard. Examination of such low-level image attributes on saccadic performance is an intriguing area for further testing.

Finally, we have shown the degree to which manipulations in the size of the central distractor can significantly affect saccadic latency (expt. 4). Each unit of increase, in which the patch doubled in size, was accompanied by a steady decrease in latency. This effect was nearly identical when we filtered out the high-contrast edges of the patches, so it is unlikely to be attributable to such edges stimulating increasingly eccentric locations along an extended fixation zone (Walker et al. 1997). We proposed that as the size of the texture increased it was no longer interpreted by the visual system as a salient element (such as a figure or an object) in the visual field. Another possibility mentioned earlier, although perhaps not mutually exclusive from the previous suggestion, is that as patch size increased, its ability to drive collicular fixation neurons decreased. This is not necessarily inconsistent with an extended fixate system. Findlay and Walker (1999) noted that their idea of such a system was not meant to imply that the receptive fields of fixation neurons extended $10 \mathrm{deg}$, only that the distributed network of these cells might extend to this extreme. Our results do support the idea that the foveal region is particularly sensitive to nontarget stimulation, provided the stimulus is small enough to act as a fixation target. What is less clear is whether the effect for peripheral distractors reflects an extended fixation region, or whether such a result is better explained by the competitive interaction mechanism suggested by Olivier et al. (1999). We believe our results, in particular the lack of a target $\times$ distractor interaction in expt. 1, suggest the latter explanation.

We realize that we have limited this discussion to the SC at the expense of oversimplification. It has been known for some time that the SC receives signals from various cortical areas (e.g., frontal eye fields: Segraves and Goldberg 1987; lateral intraparietal area: Lynch et al. 1985). It is also believed that the $\mathrm{SC}$ is an important structure for integrating these signals (Trappenberg et al. 2001) before sending a final message to the saccade generating circuitry in the brain stem, but it is beyond the scope of this paper to speculate on how these signals might contribute to the effects reported here. Our emphasis on the SC was purely motivated by its presumed role in the remote distractor effect (Findlay and Walker 1999; Olivier et al. 1999; 
Trappenberg et al. 2001; Walker et al. 1997), and other inhibitory effects on saccadic programming (Baccino et al. 2001; Reingold and Stampe 2000, 2002). The series of experiments reported here should provoke more questions as to the nature of the mechanisms believed to underlie the effect of nontarget stimuli on a saccadic response.

Our results might also have implications for higher-level figure-ground processes on saccadic eye movements. In particular, it brings up the interesting question of whether neurons in the fixation region of the SC already possess the capability of distinguishing figure from ground, or whether such processing is mediated only by cortical areas (e.g., V1; Lamme 1995). Further research is needed to explore this intriguing question.

\section{A C K N OW LEDGMENTS}

We gratefully acknowledge the helpful comments of D. Braun and four anonymous reviewers.

\section{G R A N T S}

This research was funded by the Bundesministerium für Bildung und Forschung (Project ModKog). D. Kerzel was supported by the Deutsche Forschungsgemeinschaft (DFG KE 825/3-1 and 825/4-1,2).

\section{REFERENCES}

Baccino T, Wolfgang $\mathbf{J}$, and Bussolon $\mathbf{J}$. The influence of bright background flicker during different saccade periods on saccadic performance. Vision Res 41: 3909-3916, 2001.

Braun D and Breitmeyer B. Effects of reappearance of fixated and attended stimuli upon saccadic reaction time. Exp Brain Res 81: 318-324, 1990.

Burton GJ and Moorhead IR. Color and spatial structure in natural scenes. Appl Opt 26: 157-170, 1987.

Deubel H, Wolf W, and Hauske M. The evaluation of the oculomotor error signal. In: Theoretical and Applied Aspects of Oculomotor Research, edited by Gale AG and Johnson FW. Amsterdam: Elsevier, 1984.

Dorris MC and Munoz DP. Saccadic probability influences motor preparation signals and time to saccadic initiation. J Neurosci 18, 7015-7026, 1998.

Dorris MC, Paré M, and Munoz DP. Neuronal activity in monkey superior colliculus related to the initiation of saccadic eye movements. J Neurosci 17: 8566-8579, 1997.

Fendrich R, Demirel S, and Danziger S. The oculomotor gap effect without a foveal fixation point. Vision Res 39: 833-841, 1999.

Findlay JM. Global processing for saccadic eye movements. Vision Res 22: 1033-1045, 1982.

Findlay JM and Walker R. A model of saccade generation based on parallel processing and competitive inhibition. Behav Brain Sci 22: 661-721, 1999.

Fischer B and Ramsperger E. Human express saccades: extremely short reaction times of goal directed eye movements. Exp Brain Res 57: 191-195, 1984.

Gandhi NJ and Keller EL. Spatial distribution and discharge characteristics of superior colliculus neurons antidromically activated from the omnipause region in monkey. J Neurophysiol 78: 2221-2225, 1997.

Kingstone A and Klein RM. Visual offsets facilitate saccadic latency: does predisengagement of visuospatial attention mediate this gap effect? $J$ Exp Psychol Hum Percept Perform 19: 1251-1265, 1993.

Knox PC and Bekkour T. Spatial mapping of the remote distractor effect on smooth pursuit initiation. Exp Brain Res 154: 494-503, 2004.

Lamme VA. The neurophysiology of figure-ground segregation in primary visual cortex. J Neurosci 15: 1605-1615, 1995.

Lévy-Schoen A. Determination et latence de la response oculomotrice a deux stimulus. Ann Psychol 74: 43-66, 1969.

Lynch JC, Graybiel AM, and Lobeck LJ. The differential projection of two cytoarchitectonic subregions of the inferior parietal lobule of macaque upon the deep layers of the superior colliculus. J Comp Neurol 235: 241-254, 1985.

Macmillan NA and Creelman CD. Detection Theory: A User's Guide. New York: Cambridge Univ. Press, 1991.
McPeek RM and Schiller PH. The effects of visual scene composition on the latency of saccadic eye movements of the rhesus monkey. Vision Res 34: 2293-2305, 1994.

Munoz DP and Istvan PJ. Lateral inhibitory interactions in the intermediate layers of the monkey superior colliculus. J Neurophysiol 79: 1193-1209, 1998.

Munoz DP and Wurtz RH. Fixation cells in monkey superior colliculus. I. Characteristics of cell discharge. J Neurophysiol 70: 559-575, 1993a.

Munoz DP and Wurtz RH. Fixation cells in monkey superior colliculus. II. Reversible activation and deactivation. J Neurophysiol 70: 576-589, 1993 b.

Munoz DP and Wurtz RH. Saccade-related activity in monkey superior colliculus. I. Characteristics of burst and buildup cells. J Neurophysiol 73: 2313-2333, 1995a.

Munoz DP and Wurtz RH. Saccade-related activity in monkey superior colliculus. II. Spread of activity during saccades. J Neurophysiol 73: 2334-2348, 1995b

Olivier E, Dorris MC, and Munoz DP. Lateral interactions in the superior colliculus, not an extended fixation zone, can account for the remote distractor effect. Commentary on J. M. Findlay and R. Walker. Behav Brain Sci 22: 694-695, 1999.

Párraga CA, Troscianko T, and Tolhurst DJ. The human visual system is optimized for processing the spatial information in natural visual images. Curr Biol 10: 35-38, 2000.

Reingold EM and Stampe DM. Saccadic inhibition and gaze contingent research paradigms. In: Reading as a Perceptual Process, edited by Kennedy A, Radach R, Heller D, and Pynte J. Amsterdam: Elsevier, 2000.

Reingold EM and Stampe DM. Saccadic inhibition in voluntary and reflexive saccades. J Cogn Neurosci 14: 371-388, 2002.

Reuter-Lorenz PA, Oonk HM, Barnes LL, and Hughes HC. Effects of warning signals and fixation point offsets on the latencies of pro- versus antisaccades: implications for an interpretation of the gap effect. Exp Brain Res 103: 287-293, 1995.

Rizzolatti G, Buchtel HA, Camarda R, and Scandolara C. Neurons with complex visual properties in the superior colliculus of the macaque monkey. Exp Brain Res 38: 37-42, 1980.

Ross LE and Ross SM. Saccade latency and warning signals: stimulus onset, offset, and change as warning events. Percept Psychophys 27: 251-257, 1980.

Saslow MG. Effects of components of displacement-step stimuli upon latency for saccadic eye movement. J Opt Soc Am 57: 1024-1029, 1967.

Schiller PH, Haushofer J, and Kendall G. How do target predictability and precueing affect the production of express saccades in monkeys? Eur J Neurosci 19: 1963-1968, 2004.

Segraves MA and Goldberg ME. Functional properties of corticotectal neurons in the monkey's frontal eye field. J Neurophysiol 58: 1387-1419, 1987.

Tam WJ. Ocular disengagement inhibited by target onset in periphery? Commentary on J. M. Findlay and R. Walker. Behav Brain Sci 22: 698, 1999.

Thorpe SJ, Gegenfurtner KR, Fabre-Thorpe M, and Bülthoff HH. Detection of animals in natural images using far peripheral vision. Eur J Neurosci 14: 869-876, 2001.

Trappenberg TP, Dorris MC, Munoz DP, and Klein RM. A model of saccade initiation based on the competitive integration of exogenous and endogenous signals in the superior colliculus. J Cogn Neurosci 13: $256-$ $271,2001$.

Vergilino-Perez D and Findlay JM. Foveal stimulation and saccadic latencies. Exp Brain Res 150: 255-258, 2003.

Walker R, Deubel H, Schneider WX, and Findlay JM. Effect of remote distractors on saccade programming: evidence for an extended fixation zone. J Neurophysiol 78: 1108-1119, 1997.

Walker R, Kentridge RW, and Findlay JM. Independent contributions of the orienting of attention, fixation offset, and bilateral stimulation on human saccadic latency. Exp Brain Res 103: 294-310, 1995.

Weber H, Aiple F, Fischer B, and Latanov A. Dead zone for express saccades. Exp Brain Res 89: 214-222, 1992.

Weber $\mathbf{H}$ and Fischer B. Differential effects of non-target stimuli on the occurrence of express saccades in man. Vision Res 34: 1883-1891, 1994.

Wenban-Smith MG and Findlay JM. Express saccades: is there a separate population in humans? Exp Brain Res 87: 218-222, 1991. 